\title{
金属氧化物催化生物质衍生羧酸酮基化研究进展
}

\author{
丁爽葛庆峰祝新利* \\ (天津大学化工学院 天津化学化工协同创新中心 天津 300072)
}

\begin{abstract}
摘要 从可再生的木质纤维素生物质制备液体燃料受到越来越多的关注. 有机羧酸是生物质解聚生物油的重要成分, 使得生物油具有酸性、腐蚀性和不稳定性. 因而, 羧酸的去除十分关键. 酮基化反应将两分子羧酸转化为酮、二氧化碳 和水, 不使用氢气的情况下高效脱氧且增加碳链长度. 此外, 生成的酮为重要化学品. 目前酮基化反应的机理和活性 位的研究还存在争论. 因酮基化反应过程生成的中间产物不同(如 $\beta$-䣳酸、酮烯、羧化物、酰基碳正离子等), 研究者们 提出了不同的反应机理, 如 $\beta$-酮酸机理和酮烯机理. 酮基化反应属于结构敏感性反应, 因此金属氧化物表面结构的不 同会导致酮基化反应活性不同. 酸碱位协同作用在酮基化反应过程中是必不可少的，同时氧空位可以提高酮基化反应 的活性. 本综述重点从酮基化反应机理、金属氧化物的表面结构、酸碱性及氧化还原性方面对酮基化反应进行了评述, 并对其进行了展望.
\end{abstract}

关键词 羧酸; 酮基化; 金属氧化物; 表面结构及性质; 反应机理

\section{Research Progress in Ketonization of Biomass-derived Carboxylic Acids over Metal Oxides}

\author{
Ding, Shuang Ge, Qingfeng Zhu, Xinli* \\ (Collaborative Innovation Center of Chemical Science and Engineering, School of Chemical Engineering and \\ Technology, Tianjin University, Tianjin 300072, China)
}

\begin{abstract}
With the increasing needs for transportable fuels and the growing concerns on environmental pollution, significant attention has been paid to the conversion of renewable lignocellulosic biomass to liquid fuels. As a major component of bio-oil from biomass depolymerization, organic carboxylic acids make the bio-oil acidic, corrosive and unstable, which are harmful for storage, transportation, and upgrading of bio-oil. Therefore, the removal of carboxylic acids is very important. Ketonization reaction, also called ketonic decarboxylation, converts two moles carboxylic acids to ketone (symmetrical or asymmetrical ketones), carbon dioxide and water, which removes oxygen efficiently and increases the carbon chain length without using hydrogen. In addition, ketones are important chemicals and have been widely used in chemical industry as organic solvent. The mechanism and active site for ketonization are still under debate. Various mechanisms have been proposed for the ketonization, based on different reaction intermediates evolved (i.e., $\beta$-keto-acids, ketene, carboxylates and acyl carbonium ions). Ketonization reaction is a surface-structure-sensitive reaction, thus reaction activity depends on surface-structure of the metal oxides (such as crystal surfaces and particle size). The concerted function of oxygen anions (Brønsted bases) and unsaturated metal cations (Lewis acids) is crucial for ketonization. The amphoteric oxides show better catalytic activity than pure acidic or basic oxides. Oxygen vacancy formed on the surface of metal oxides is a key factor for high ketonization activity, which can stabilize the reaction product and reduce the activation energy. This paper reviews the progress in ketonization from the aspects of reaction mechanism, and the effects of surface structure, acidity and basicity, and reducibility of metal oxides on ketonization. The $\beta$-keto-acids based mechanism and ketene based mechanism will be discussed in detail to understand how does the $\mathrm{C}-\mathrm{C}$ coupling happen and the fundamental role of $\alpha-\mathrm{H}$. Finally, the importance of surface structure and properties of metal oxides on the carboxylic acids ketonization reaction is explained.
\end{abstract}

Keywords carboxylic acid; ketonization; metal oxides; surface-structure and property; reaction mechanism

\section{1 引言}

随着经济的快速发展和人口的不断增长, 对能源的 需求越来越多. 煤、石油、天然气这些非可再生能源的 日益消耗, 不仅造成了环境污染, 全球气候变暖等问题,
而且也带来了能源危机等一系列严重后果. 生物质能源 是一种可再生的清洁资源, 不仅储量丰富而且分布范围 广泛. 生物质资源的开发和利用已经得到越来越多的关 注 $^{[1 \sim 6]}$. 不同的化学方法可以将生物质转化成含 $\mathrm{C} 、 \mathrm{H}$ 、 $\mathrm{O}$ 的有机化合物(如醇、醛、羧酸、酚、芳香烃类等小分

*E-mail: xinlizhu@tju.edu.cn

Received February 16, 2017; published April 7, 2017.

Project supported by the National Natural Science Foundation of China (Grant Nos. 21676194 and 21373148) and the Ministry of Education of China Program of New Century Excellent Talents in University (Grant No. NCET-12-0407).

项目受国家自然科学基金(Nos. 21676194 和 21373148)和教育部新世纪优秀人才支持计划(NCET-12-0407)资助. 
子含氧有机化合物), 最常用的方法是快速热解和水解 反应 ${ }^{[7]}$. 这些小分子有机化合物还可以通过进一步转化 生成汽油、柴油和其他化学品 ${ }^{[1]}$. 生物油主要是由木质 素转化生成的, 羧酸是生物油中的重要组成部分. Sedran 等 $^{[8]}$ 对生物油中的组成进行了测定, 羧酸的含量 高达 $17 \%$. 羧酸具有不稳定性, 容易发生聚合生成大分 子物质, 且具有腐蚀性能损坏反应设备及管路, 所以羧 酸的进一步转化是首先要解决的问题. 羧酸可以通过选 择性加氢生成醇或醛, 还可以通过酮基化反应生成酮.

酮基化反应转化羧酸具有如下优点. 第一, 反应绿 色无污染, 并且不需要溶剂和其他试剂; 第二, 反应不 需要氢气就可以将有机物中的氧移除; 第三, 反应所需 要的催化剂一般都是低成本的; 第四, 在转化掉高活性 的羧基的同时又增长了有机物碳链的长度, 生成了更稳 定的化合物. 生成的酮类化合物可以继续进行羟醛缩合 反应，进一步转化成其他有机大分子化合物.

酮基化反应(也称脱羧酮基化反应)是将两分子的羧 酸脱去一个羰基生成酮、二氧化碳和水, 如反应式(1)所 示. 相同的两个羧酸分子 $\left(\mathrm{R}^{1}=\mathrm{R}^{2}\right)$ 进行酮基化反应，称 为自身酮基化; 两个不同的羒酸分子 $\left(\mathrm{R}^{1} \neq \mathrm{R}^{2}\right)$ 进行反应 则称为交叉酮基化 ${ }^{[015]}$. Sato 等 ${ }^{[10]}$ 报道了丙酸与其它羧 酸反应, 生成物中包括一种不对称的酮和两种对称的 酮, 生成物的组成遵循二项式分布.

$$
\mathrm{R}^{1} \mathrm{COOH}+\mathrm{R}^{2} \mathrm{COOH} \rightarrow \mathrm{R}^{1} \mathrm{COR}^{2}+\mathrm{CO}_{2}+\mathrm{H}_{2} \mathrm{O}
$$

早在 1858 年, Friedel ${ }^{[16]}$ 首次报道了酮基化反应, 通 过醋酸钙裂解来制备丙酮. 直到第一次世界大战前, 该 过程被用来工业上制备丙酮. 经过多年的研究, 研究者 逐步开发出金属氧化物、沸石分子篮等催化剂, 直接催 化羧酸酮基化反应生成酮. 目前工业上利用氧化锆、氧 化铝负载型催化剂催化羧酸酮基化反应, 制成的酮化学 品是甲基异丁基酮(MIPK). 在高温催化剂作用下, 异丁 酸和乙酸进行交叉酮基化反应生成 MIPK. 德国康得阿 维斯塔公司通过异丁酸与乙酸酮基化反应生成 MIPK, 每年生产能力达 $1500 \mathrm{t}^{[17]}$. 国内的吉化公司化肥厂通过 氧化锆催化异丁酸与乙酸反应制备酮的年生产能力达 到 $250 \mathrm{t}^{[17]}$, 同时北京化工研究院自 1998 年自行开发了 异丁酸与乙酸合成 MIPK技术, 建立了每年 $300 \mathrm{t}$ 的小规 模装置. 虽然金属氧化物催化羧酸酮基化反应在工业上 有成功的应用，但仍存在着反应温度高、催化剂活性低、 积炭造成的稳定性差等问题. 这些问题的解决需要从机 理上更好地认知酮基化反应.

针对羧酸酮基化反应, 在理论和实验研究过程中, 研究者们分别从 $\alpha-\mathrm{H}$ 的作用 ${ }^{[10,18]}$ 、取代基空间位阻 ${ }^{[9,10]}$ 、 羧酸碳链长度 ${ }^{[10,19]}$ 、中间产物 ${ }^{[20,21]}$ 、催化剂种类 ${ }^{[22 ~ 28] 、 ~}$ 反应机理 ${ }^{[29 \sim 31]}$ 及动力学 ${ }^{[32 ~ 34]}$ 上做了不同程度探索. 但 对于催化剂活性位、反应机理、 $\alpha-\mathrm{H}$ 的作用仍存在着一 定的争论. 与此同时, 也有研究者对酮基化反应进行过

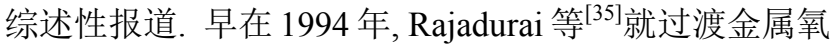
化物催化羧酸反应路径进行了系统的整理, 通过对中间 物种类和动力学数据分析提出了羧酸酮基化反应的酮 烯机理. Renz 等 ${ }^{[36]}$ 也从机理方面综述了羧酸酮基化反 应, 文中指出酮基化反应中弱碱可以提高催化活性. 宁 朋歌等 ${ }^{[37]}$ 从催化剂、反应物、反应温度和反应机理方面 介绍了羧酸气相酮基化反应过程, 综述了国内外的研究 进展, 并探讨了未来的发展方向. 2013 年, Resasco 等 ${ }^{[38]}$ 从反应机理、催化剂和酮基化在生物质转化中的应用三 方面进行了系统的报道. Pacchioni 等 ${ }^{[39]}$ 从密度泛函理论 (DFT)方面介绍了氧化钣和氧化锆催化羧酸酮基化反应.

不同于之前的综述报道, 本文重点从金属氧化物催 化羧酸酮基化反应, 介绍氧化物催化反应机理和催化剂 结构性质对酮基化反应的影响这两方面进行论述.

\section{2 金属氧化物催化的酮基化反应机理}

尽管酮基化反应经历了很长时间的研究, 但到目前 对于酮基化反应机理的认识还没有达成统一. 酮基化反 应分为体相酮基化反应和表面酮基化反应，也可以通过 生成的中间产物不同将反应机理分为酮烯机理和 $\beta$-酮 酸机理.

\section{1 体相与表面酮基化反应}

体相酮基化反应是羒酸盐经过裂解生成酮, 具有低 晶格能量的氧化物会发生体相反应(如碱金属和碱土金 属); 表面酮基化反应则是吸附在催化剂表面的羧化物 在 $\alpha-\mathrm{H}$ 的作用下形成不同的中间产物，进而脱羧生成 酮, 具有高晶格能量的氧化物发生表面酮基化反应(如 氧化钛、氧化铈、氧化锆、氧化锰 $)^{[38]}$. 虽然能根据金属 氧化物催化剂具有的晶格能量将反应区分为体相进行 和表面进行，但是否经过体相或是表面反应，不仅与金 属氧化物具有的晶格能量有关, 还与反应温度有关.

Zaki 等 ${ }^{[40]}$ 对氧化镁 $(\mathrm{MgO})$ 研究发现, 虽然 $\mathrm{MgO}$ 具 有高晶格能量, 也能进行体相酮基化反应, 这取决于反 应温度高低. 由于 $\mathrm{MgO}$ 具有强碱性, 在低温条件下, 乙 酸分子吸附到催化剂表面形成体相的乙酸镁, 温度高于 $225{ }^{\circ} \mathrm{C}$ 后, 乙酸镁发生热解反应生成丙酮、 $\mathrm{CO}_{2}$ 和 $\mathrm{H}_{2} \mathrm{O}$. 当温度高于 $300{ }^{\circ} \mathrm{C}$ 后, 乙酸在 $\mathrm{MgO}$ 表面活化经过表面 酮基化反应生成丙酮、 $\mathrm{CO}_{2}$ 和 $\mathrm{H}_{2} \mathrm{O}$. Shanks 等 ${ }^{[31]}$ 对氧化 铈 $\left(\mathrm{CeO}_{2}\right)$ 催化羒酸酮基化反应也做了相同的研究. 不管 是液相反应还是气相反应, $\mathrm{CeO}_{2}$ 催化羧酸反应根据反应 温度的高低也可以分别进行表面和体相反应. 在 150 $300{ }^{\circ} \mathrm{C}$ 之间, $\mathrm{CeO}_{2}$ 催化羧酸反应是经过体相转化的, 也 就是先生成体相羧酸盐, 然后经过热解生成酩、 $\mathrm{CO}_{2}$ 和 $\mathrm{H}_{2} \mathrm{O}$. Sato 等 ${ }^{[27]}$ 研究了稀土氧化物(氧化镧、氧化铈、氧 化镨和氧化钕)催化乙酸酮基化反应, 反应过程中也经 过体相转化. 体相酮基化反应是通过形成体相羧酸盐发 生热解生成酮, 而表面酮基化反应则是在催化剂表面进 
行. 通过在催化剂表面生成的中间物不同又可将酮基化 反应机理划分为不同的反应机理, 在 2.2 和 2.3 节将详细 介绍两种表面催化酮基化反应机理.

\section{$2.2 \beta$-酮酸机理}

$\beta$-酮酸机理是在反应过程中, 两分子羧酸先生成 $\beta$ 酮酸中间产物, 然后再继续转化形成酮, 如图 1 所示, 以双配位吸附方式为例.
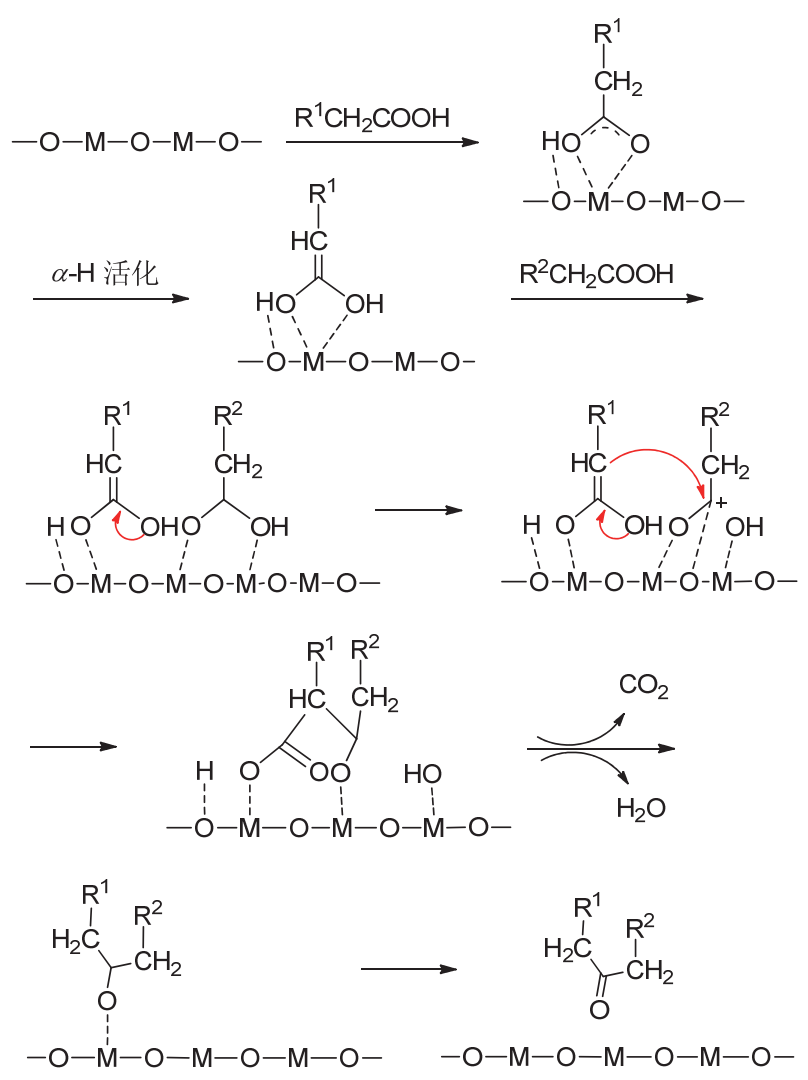

图 1 金属氧化物催化羧酸酮基化反应机理 $(\beta$-酮酸机理)示意图

Figure $1 \beta$-ketoacid based mechanism for ketonization of carboxylic acid over metal oxide

在此反应过程中, 第一个羧酸分子吸附到催化剂表 面，经去质子化后，羧基的氧原子吸附在金属阳离子上， 而氢原子吸附在表面的氧原子上, 然后 $\alpha-\mathrm{H}$ 经表面的氧 原子活化形成羧酸的阴离子或经过烯醇化形成中间物. 第二个羧酸分子吸附时, 羧基的氧原子吸附在金属阳离 子上, 然后羧基中的碳-差基键 $(\mathrm{C}-\mathrm{OH}$ 键)断裂, 形成 乙酰基碳正阳离子. 第一个羧酸变形后的中间物亲核进 攻酰阳离子, 形成 $\beta$-酮酸, 然后碳-碳键 $(\mathrm{C}-\mathrm{C}$ 键 $)$ 断裂, 脱去一分子 $\mathrm{CO}_{2}$, 形成丙酮; 同时羟基和氢原子键合, 脱去一分子水. 金属阳离子吸附羧化物的形式有多种, 单配位吸附、桥连吸附和螯合配位吸附.

Pulido 等 ${ }^{[29]}$ 通过实验数据并结合 DFT 计算对比氧 化锆 $\left(\mathrm{ZrO}_{2}\right)$ 催化乙酸酮基化反应, 此反应可经过 $\beta$-酮酸 过程和直接转化过程, 如图 2 所示. 在 $\beta$-酮酸过程中,
两分子乙酸吸附在相邻的活性位上，一分子乙酸形成烯 醇化合物, 另一分子乙酸则形成酰阳离子, 然后烯醇化 合物进攻酰阳离子生成 $\beta$-酮酸中间产物, 然后经过 $\mathrm{C}-$ $\mathrm{C}$ 键断裂形成丙酮并脱除 $\mathrm{CO}_{2}$ 和 $\mathrm{H}_{2} \mathrm{O}$. 而在直接转化的 路径中, 一分子乙酸盐直接进攻另一分子乙酸, 生成丙 酮. 经过 $\beta$-酮酸机理的活化能 $(108 \mathrm{~kJ} / \mathrm{mol})$ 要低于直接 转化的活化能 $(154 \mathrm{~kJ} / \mathrm{mol})$, 在动力学上更支持 $\beta$-酮酸 机理. Resasco 等 ${ }^{[41]}$ 报道了 $\mathrm{Ru} / \mathrm{TiO}_{2} / \mathrm{C}$ 催化乙酸发生液相 酮基化反应中经过 $\beta$-酮酸机理, 如图 3 所示. 在反应之 前, 氧化钛先经过 $\mathrm{H}_{2}$ 预处理, 使 $\mathrm{Ti}^{4+}$ 被还原成 $\mathrm{Ti}^{3+}$ 的同 时, 表面形成羟基(-OH), 然后进行酮基化反应. 在反应 中, 一分子乙酸羧基中 $\mathrm{H}$ 与表面羟基键合脱水，同时乙 酸盐中的两个氧原子以桥联的方式吸附在阳离子上, 然 后在 $\alpha-\mathrm{H}$ 作用下形成烯醇化合物. 之后第二分子乙酸着 基中的氧原子进攻烯醇化合物中的 $\mathrm{H}$, 形成 $\beta$-酮酸中间 产物, 再经过 $\mathrm{C}-\mathrm{C}$ 键断裂形成丙酮和 $\mathrm{CO}_{2}$. 虽然二者 都提出了 $\beta$-酮酸机理, 但因为所用催化剂的酸碱性、氧 化还原性和表面结构不同导致具体的反应细节不同. 在 氧化锆的表面，乙酸可以经过脱羟基形成酰阳离子，而 在氧化钛表面则没有形成. 目前大多数的研究者认为羧 酸酮基化反应遵循 $\beta$-酮酸机理 ${ }^{[9,15,19,29 ~ 31]}$.

a) $\beta$-keto-acid route

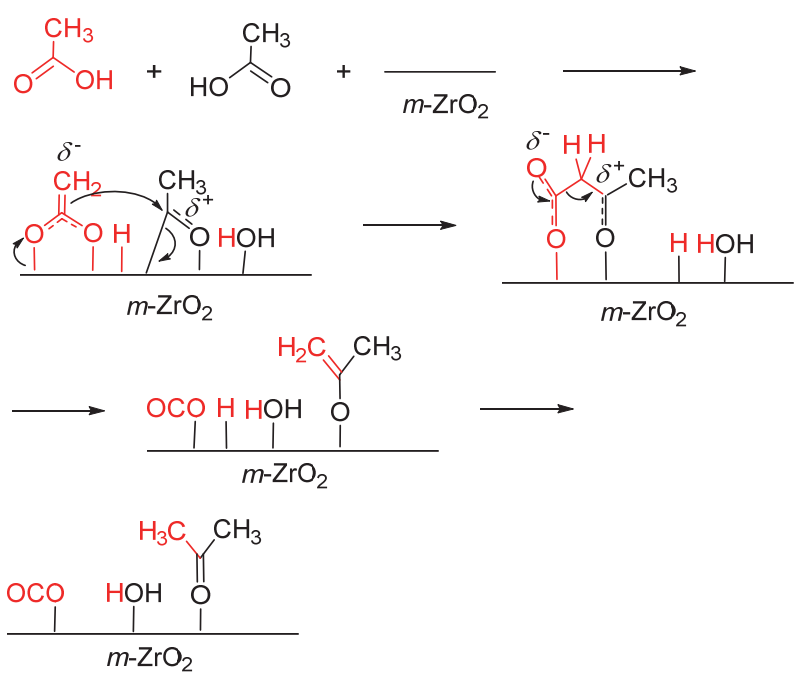

b) Concerted route

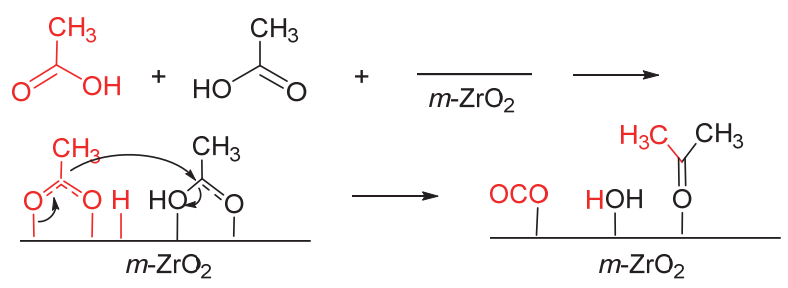

图 $2 \mathrm{~m}-\mathrm{ZrO}_{2}$ 催化乙酸脱羧酮基化反应机理 ${ }^{[29]}$

Figure 2 Reaction mechanism for acetic acid decarboxylation over $m-\mathrm{ZrO}_{2}{ }^{[29]}$ 


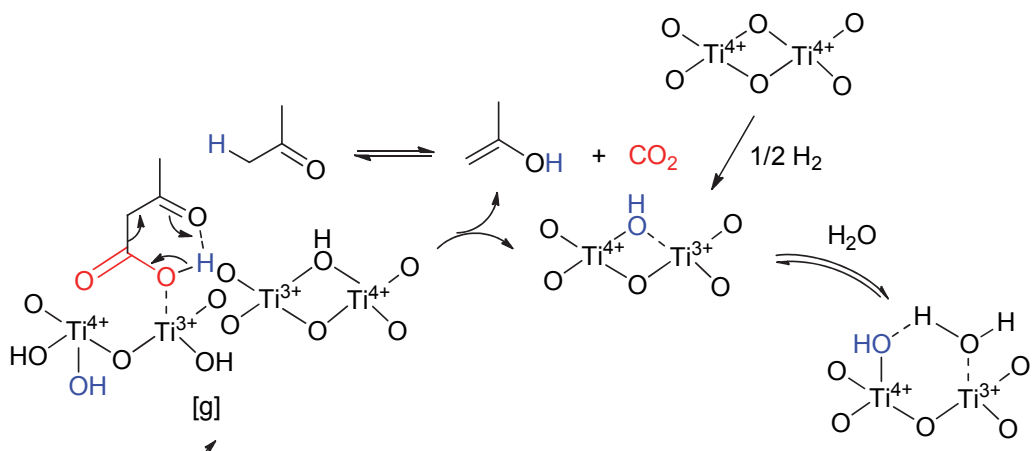<smiles></smiles>

[a]<smiles>CC(C)C(C)CO</smiles><smiles></smiles>

[b]<smiles>CCC(C)CO</smiles>

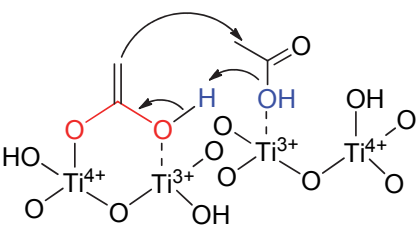

[e]<smiles>CC(C)C</smiles><smiles>C=C1O[N+](C)(O)O[I+](O)(O)O1</smiles>

[d]

图 $3 \mathrm{TiO}_{2}$ 催化乙酸液相反应 $\beta$-酮酸机理 ${ }^{[41]}$

Figure 3 Proposed $\beta$-ketoacid based mechanism for liquid phase ketonization of acetic acid over $\mathrm{TiO}_{2}{ }^{[4]}$

\section{3 酮烯机理}

除了 $\beta$-酮酸机理外, 也有研究者提出酮烯机理, 但 只有具有 $\alpha-\mathrm{H}$ 的羧酸才能经过此过程. 酮烯机理就是单 分子羧酸先进行脱水生成酮烯 $\left(\mathrm{R}_{2} \mathrm{C}=\mathrm{C}=\mathrm{O}\right)$, 然后再进 行 $\mathrm{C}-\mathrm{C}$ 耦合反应, 如图 4 所示.

在此反应过程中, 吸附的羧酸分子先进行单分子脱 水, 生成酮烯; 然后酮烯直接和羧化物反应生成丙酮和 $\mathrm{CO}_{2}$, 或者酮烯转变成乙酰基碳正离子再与羧化物反应 生成丙酮和 $\mathrm{CO}_{2}$. 酰阳离子的形成与氧化物的路易斯酸 性有关, 酸性强则有利于形成酰阳离子.

Rajadurai 等 ${ }^{[35]}$ 研究认为羧酸进行酮基化反应是经 过酮烯反应机理, 反应式如(2)所示. 当反应温度高于 $400{ }^{\circ} \mathrm{C}$ 时, 吸附的两分子乙酸, 先进行解离生成羒化物 $\left(\mathrm{CH}_{3} \mathrm{COO}^{-}\right)$和氢离子 $\left(\mathrm{H}^{+}\right)$, 然后直接进行酮基化反应; 当反应温度低于 $400{ }^{\circ} \mathrm{C}$ 时, 经过酮烯中间产物生成丙 酮. Barteau 等 ${ }^{[42]}$ 使用 $\mathrm{Pd} / \mathrm{CeO}_{2}$ 和 $\mathrm{Co} / \mathrm{CeO}_{2}$ 低温催化酮

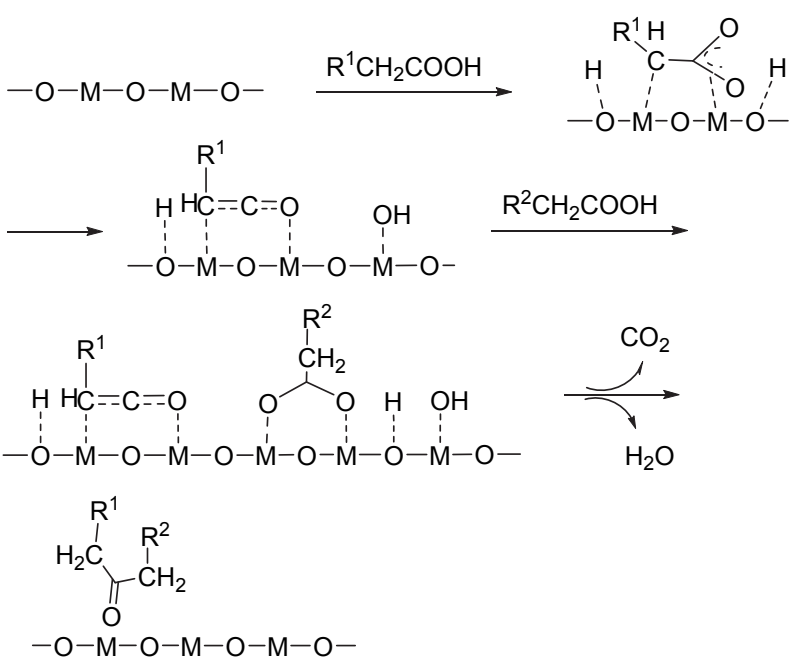

图 4 金属氧化物催化着酸酮基化反应机理(酮烯机理)示意图 Figure 4 Ketene-based mechanism of carboxylic acid ketonization over metal oxide 


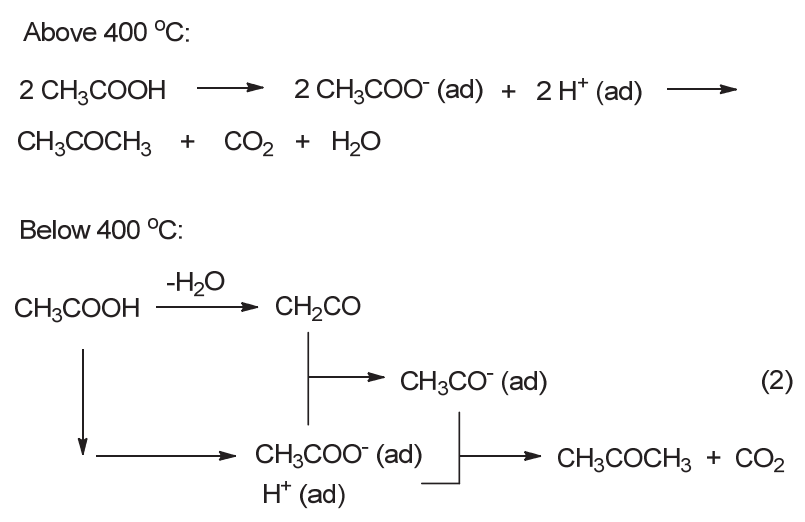

基化反应，中间过程就是乙酰基参与反应生成酮. Dooley 等 ${ }^{[12]}$ 通过对 $\mathrm{CeO}_{2}$ 催化酸-酸、酸-醛的交叉酮基 化动力学研究, 采用同位素标记的方法测定中间物, 认 为此交叉酮基化反应是经过酮烯机理生成酮. 而 Ponec 等 ${ }^{[18]}$ 则认为酮烯只是一个副产物而不是中间产物.

\section{3 酮基化金属氧化物催化剂}

用于催化酮基化反应的催化剂种类繁多, 有金属氧

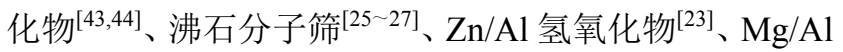
水滑石 ${ }^{[24]}$ 等. 本文聚焦探讨金属氧化物对酮基化反应 的影响, 包括催化剂结构、酸碱性、氧化还原性等对反 应活性的影响. 在过去的几十年里, 开发了大量的金属 氧化物用于催化羧酸酮基化反应. 2014 年, Glinski 等 ${ }^{[45]}$ 报道了 32 种元素氧化物对丙酸酮基化反应活性的影响. 通过活性测定将氧化物分成三组: 低活性催化剂、中等 水平活性催化剂、高活性催化剂, 具有高活性的元素包 括锰、铅、铈、针和铀. 除了这些负载型的氧化物(载体 一般为二氧化硅、三氧化二铝和氧化钛), 混合氧化物 (如 $\mathrm{CeZrO}_{x}{ }^{[46]}, \mathrm{Zn}_{x} \mathrm{Zr}_{y} \mathrm{O}_{z}^{[47]}, \mathrm{CeMnO}_{x}{ }^{[48]}$ 和 $\mathrm{Zr}-\mathrm{Mg}-\mathrm{Y}-\mathrm{O}^{[49]}$ 等)同样具有较高的催化活性. 作者采用红外光谱(IR)、 程序升温还原(TPR)、程序升温脱附(TPD)、比表面积测 定(BET)、X 衍射光谱(XRD)等不同的技术表征手段探究 催化剂的活性位、结构、酸碱性、氧化还原性对反应活 性的影响. 本文将从表面结构、酸碱性、氧化还原性这 三方面系统介绍金属氧化物催化剂对羧酸酮基化反应 的影响.

\section{1 催化剂表面结构影响}

多相催化反应可以分为两类 ${ }^{[50]}$ : 一是表面结构敏 感性反应, 催化剂的活性取决于催化剂的表面结构, 如 晶粒的某一晶面; 二是结构非敏感性反应, 催化剂的活 性位与表面结构无关, 此类反应活性可能与催化剂的比 表面积有关. 对于羧酸酮基化反应, 研究者们发现催化 剂的表面结构对反应活性有一定的影响, 如暴露晶面中 阴阳离子的配位数.

因其高效的储氧能力和稳定的表面结构, $\mathrm{CeO}_{2}$ 作为 催化材料被广泛应用于多种有机反应中, 如 $\mathrm{CO}$ 氧化 ${ }^{[51]}$,
水煤气变换, $\mathrm{C}-\mathrm{C}$ 耦合反应等. 在羧酸酮基化反应中, 也被广泛研究. $\mathrm{CeO}_{2}$ 具有萤石结构, 四价铈阳离子 $\left(\mathrm{Ce}^{4+}\right)$ 与八个氧负离子 $\left(\mathrm{O}^{2-}\right)$ 配位, 具有多种晶面 ${ }^{[52]}$. 在 $\mathrm{CeO}_{2}$ 的(111)、(110)和(100)晶面中, (111)晶面最稳定, 也 只有(111)晶面能够催化羒酸酮基化反应 ${ }^{[53]}$. (111) 晶面是 六方对称结构, 最外层是 $\mathrm{O}^{2-}$ 离子, 第二层是 $\mathrm{Ce}^{4+}$ 阳离 子, 同时暴露在表面, 每一个阳离子和阴离子都有一个 空位. (100)晶面是四方对称结构, 阴阳离子同时暴露在 表面, $\mathrm{O}^{2-}$ 离子是完全配位, $\mathrm{Ce}^{4+}$ 阳离子则具有四个空位. 当阴阳离子同时暴露在表面时，且金属阳离子具有不饱 和配位, 才能吸附解离后的羧化物, 进而经过一系列的 转化脱羧生成酮. 通过调变催化剂合成方法和焙烧温度 等促使形成不同的晶面和不同的颗粒尺寸, 从而控制羒 酸酮基化反应的活性.

早在 1996 年, Vohs 等 ${ }^{[33]}$ 报道了 $\mathrm{CeO}_{2}$ 不同晶面对羧 酸酮基化反应的影响. 研究结果显示, 只有(111)晶面上 能生成酮. 结合红外数据, 证明乙酸和甲酸以单配位的 形式吸附在 $\mathrm{Ce}^{4+}$, 而(100)晶面存在较多的空位不利于 发生酮基化反应. 通过改变催化剂合成条件, 可以控制 催化剂的颗粒尺寸. Wang 等 ${ }^{[54]}$ 研究了通过控制水热合 成条件调变 $\mathrm{CeO}_{2}$ 的颗粒尺寸, 当颗粒大小为 $3 \sim 10 \mathrm{~nm}$ 时, 主要是(111) 晶面和(100)晶面. 随着颗粒的增大, (100)晶面逐渐消失, 而(111)晶面增多, 催化活性增强. 焙烧温度同样会影响 $\mathrm{CeO}_{2}$ 颗粒的形貌、结晶性和氧化 态，间接地影响了羧酸酮基化反应活性. 不同的焙烧温 度会促使形成不同的晶面. Shanks 等 ${ }^{[55}$ 做了焙烧温度对 催化活性的研究, 在 $450{ }^{\circ} \mathrm{C}$ 焙烧时形成的(100)晶面较 多, 而 $900{ }^{\circ} \mathrm{C}$ 焙烧时形成的(111)晶面则占绝大部分. 在 液相反应中 $\mathrm{CeO}_{2}$ 催化乙酸酮基化, $\mathrm{CeO}_{2}$ 的结晶性影响 催化活性. 随着焙烧温度的升高, 乙酸酮基化初始反应 速率逐渐增大, 如表 1 所示. 在 $270{ }^{\circ} \mathrm{C}$ 反应, 反应速率 从 $52 \mu \mathrm{mol} \cdot \mathrm{m}^{-2} \cdot \mathrm{h}^{-1}\left(450{ }^{\circ} \mathrm{C}\right.$ 焙烧)增大到 $267 \mu \mathrm{mol} \cdot \mathrm{m}^{-2}$. $\mathrm{h}^{-1}$ (900 ${ }^{\circ} \mathrm{C}$ 焙烧). Sato 等 ${ }^{[50]}$ 研究了在 $350{ }^{\circ} \mathrm{C}$ 丙酸发生 酮基化反应, $\mathrm{CeO}_{2}$ 颗粒尺寸能够影响反应活性, 如图 3 所示. 随着颗粒尺寸的增大, 丙酸的转化率呈现出增大 的趋势, 最高达 $49.7 \%$. 主要产物 3-戊酮的选择性都在 $99 \%$ 以上, 3-戊酮的时空收率也增加了 5 倍.

因羧酸酮基化反应活性与氧化物的表面结构息息 相关, 氧化钛 $\left(\mathrm{TiO}_{2}\right)$ 作为具有三种晶体结构的氧化物被 应用到酮基化反应中具有一定的研究意义. $\mathrm{TiO}_{2}$ 作为催 化剂被使用最多的结构是锐钣矿型和金红石型，二者因 其相异的物理、化学性质而有着不同的用途 ${ }^{[56]}$. 在这两 种晶相中, 四价钛阳离子 $\left(\mathrm{Ti}^{4+}\right)$ 与六个 $\mathrm{O}^{2-}$ 阴离子配位形 成八面体 ${ }^{[52]} . \mathrm{TiO}_{2}$ 的晶面结构和组成决定着羒酸的不同 反应路径, 不同晶面对羧酸的吸附方式不同. 在还原态 的晶面, 羧酸吸附到氧空位上, 直接进行脱氧反应. 当 晶面上的 $\mathrm{Ti}^{4+}$ 存在不饱和配位时, 则会吸附羧酸发生单 分子脱水反应或双分子酮基化反应. 
表 1 焙烧温度对初始速率的影响[5]

Table 1 Initial rate studies for the ceria catalysts ${ }^{[5]}$

\begin{tabular}{ccc}
\hline Catalyst $T_{\text {calcination }} /{ }^{\circ} \mathrm{C}$ & Initial rate $/\left(\mu \mathrm{mol} \cdot \mathrm{m}^{-2} \cdot \mathrm{h}^{-1}\right), 270{ }^{\circ} \mathrm{C}$ & Initial rate $/\left(\mu \mathrm{mol}^{\circ} \cdot \mathrm{m}^{-2} \cdot \mathrm{h}^{-1}\right), 290{ }^{\circ} \mathrm{C}$ \\
\hline 450 & 52 & 333 \\
600 & 94 & 408 \\
750 & 258 & 835 \\
900 & 267 & 1484 \\
\hline
\end{tabular}

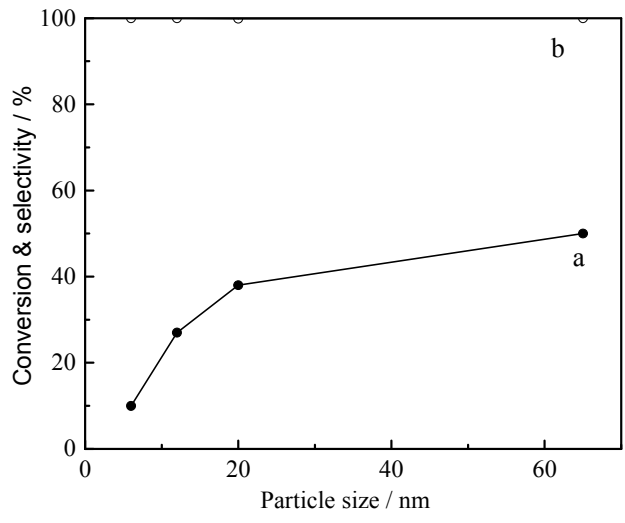

图 $5 \mathrm{CeO}_{2}$ 粒径在 $350{ }^{\circ} \mathrm{C}$ 对丙酸酮基化反应转化率和选择性的影 响 ${ }^{[50]}$. (a) 丙酸转化率, (b) 3-戊酮选择性

Figure 5 Changes in conversion and selectivity in the ketonization of propionic acid over $\mathrm{CeO}_{2}$ with different particle sizes at $350{ }^{\circ} \mathrm{C}$. (a) Conversion of propionic acid and (b) selectivity to 3-pentanone ${ }^{[50]}$

关于锐钛矿 $\mathrm{TiO}_{2}$ 单晶表面催化羧酸反应的研究, Barteau 等 ${ }^{[57]}$ 进行了最早最全面的报道, 探究羧酸反应 活性和活性位的关系. 在低温时, 乙酸以分子的形式或 是解离的形式被吸附在催化剂表面且发生脱附. 在高温 时, 乙酸盐经过三条不同的路径进行反应, 这取决于催 化剂的结构与组成. 在还原态的(001)晶面, 乙酸盐直接 进行脱氧反应. (011) 晶面暴露的 $\mathrm{Ti}^{4+}$ 与氧原子是五配位, 单分子乙酸盐吸附在此面直接进行脱水反应. (114)晶 面, 暴露的 $\mathrm{Ti}^{4+}$ 与氧原子是四配位, 双分子乙酸盐吸附 在此面进行酮基化反应生成丙酮. 在这三种晶面结构 中, 只有在(114)晶面可以进行酮基化反应, 两个乙酸分 子以单配位的形式被吸附在同一个 $\mathrm{Ti}^{4+}$ 上. Ojamäe 等 ${ }^{[58]}$ 对锐铁矿 $\mathrm{TiO}_{2}$ 纳米颗粒(110)晶面催化羧酸反应进行了 研究. 作者通过红外、拉曼光谱等表征手段, 结合 DFT 计算, 证明了去质子化后的羒酸两个氧原子吸附在两个 $\mathrm{Ti}^{4+}$ 上, 以桥连的方式存在. 催化剂表面的弛豫现象能 够影响吸附态的结构和吸附能. Resasco 等 ${ }^{[19]}$ 报道了 $\mathrm{Ru} / \mathrm{TiO}_{2}$ 催化羧酸酮基化反应动力学及机理, 也论证了 羒酸在催化剂表面以双配位的形式存在.

\section{2 催化剂酸碱性影响}

金属氧化物表面暴露的晶面类型决定了表面阴阳 离子的性质. 在金属氧化物中, 暴露的金属阳离子是路 易斯酸位( $\mathrm{L}$ 酸位), $\mathrm{O}^{2-}$ 离子则是碱性位. 金属氧化物催
化羧酸酮基化反应的活性位，一些研究者认为是碱性 位 ${ }^{[36,59]}$, 一些人认为酸性位 ${ }^{[22]}$ 也起着重要的作用. 在金 属氧化物催化羧酸酮基化反应中, 羧酸先被吸附在催化 剂表面进行解离, 羧基的 $\mathrm{H}$ 吸附在 $\mathrm{O}^{2-}$, 同时解离后的 羧化物吸附在配位不饱和金属阳离子位, 然后 $\alpha-\mathrm{H}$ 经 $\mathrm{O}^{2-}$ 活化, 羧化物经过一系列的变形后与另外一个羧化 物进行反应生成酮. 在整个反应过程中，阴阳离子均起 到了重要作用, 羧酸酮基化反应需要酸-碱活性位协同 作用.

$\operatorname{Renz}^{[36]}$ 论述了羧酸酮基化反应发生在碱性位. Zaki 等 ${ }^{[00]}$ 利用傅里叶变换红外光谱研究乙酸在 $\mathrm{Al}_{2} \mathrm{O}_{3} 、 \mathrm{CeO}_{2}$ 、 $\mathrm{TiO}_{2}$ 这三种氧化物催化下生成丙酮的反应. 因 $\mathrm{Al}_{2} \mathrm{O}_{3}$ 没 有具有可还原的 $\mathrm{L}$ 酸性位, 对丙酸没有催化活性; $\mathrm{TiO}_{2}$ 和 $\mathrm{CeO}_{2}$ 分别具有可还原的 $\mathrm{L}$ 酸性位(分别是 $\mathrm{Ti}^{4+}$ 和 $\mathrm{Ce}^{4+}$ ), 可分别在 $400{ }^{\circ} \mathrm{C}$ 和 $300{ }^{\circ} \mathrm{C}$ 催化乙酸生成丙酮、 $\mathrm{CO}_{2}$ 和水. $\mathrm{CeO}_{2}$ 的活性高于 $\mathrm{TiO}_{2}$, 是因为 $\mathrm{CeO}_{2}$ 具有更强 的碱性. $\mathrm{MgO}$ 是一种典型的碱性氧化物, 表面暴露弱酸 性位 $\left(\mathrm{Mg}^{2+}\right)$ 和碱性位 $\left(-\mathrm{OH}, \mathrm{O}^{2-}\right)$, 不仅能催化乙酸进行 表面的酮基化反应，还能使乙酸形成体相的乙酸盐，然 后经过裂解生成丙酮, $\mathrm{CO}_{2}$ 和水 ${ }^{[40]}$. 金属氧化物催化羧 酸酮基化反应，既需要碱性位活化氢，同时还需要酸性 位吸附羧化物, 这二者缺一不可.

$\mathrm{CO}_{2}$ 是一种酸性气体, 在反应中占据碱性位, 则通 入 $\mathrm{CO}_{2}$ 后反应活性会受到抑制. $\mathrm{H}_{2} \mathrm{O}$ 在催化剂表面分解 成氢和羟基, 分别占据了催化剂表面的碱性位和酸性 位，对酮基化反应活性也起到抑制作用. Dumesic 等 ${ }^{[33]}$ 以已酸为模型分子, 研究了 $\mathrm{CO}_{2}$ 和 $\mathrm{H}_{2} \mathrm{O}$ 对反应的影响, 研究结果证明了 $\mathrm{CO}_{2}$ 和 $\mathrm{H}_{2} \mathrm{O}$ 都起抑制作用. 通过 $\mathrm{NH}_{3}$-TPD 和 $\mathrm{CO}_{2}-\mathrm{TPD}$ 测定, $\mathrm{ZrO}_{2}$ 同时具有酸碱性, 且酸 性位数量 $\left(212 \mu \mathrm{mol} \cdot \mathrm{g}_{\mathrm{cat}}{ }^{-1}\right)$ 与碱性位数量相当 $(296$ $\left.\mu \mathrm{mol} \cdot \mathrm{g}_{\text {cat }}{ }^{-1}\right)$. 当以原子比 $1 / 1$ 掺杂 $\mathrm{Ce}$ 后 $\left(\mathrm{Ce}_{0.5} \mathrm{Zr}_{0.5} \mathrm{O}_{2}\right)$, 催 化剂的碱性位增多 $\left(380 \mu \mathrm{mol} \cdot \mathrm{g}_{\mathrm{cat}}{ }^{-1}\right)$, 酸性位减少 (56 $\mu$ mol $\left.\cdot \mathrm{g}_{\mathrm{cat}}{ }^{-1}\right)$. 此时 $\mathrm{Ce}_{0.5} \mathrm{Zr}_{0.5} \mathrm{O}_{2}$ 催化活性要高于 $\mathrm{ZrO}_{2}$, 碱 性位增多有利于提高酮基化反应活性.

Kijenski 等 ${ }^{[59]}$ 研究了二十种负载型氧化物催化乙酸 酮基化反应, 如表 2 所示. 在这些氧化物中, 有碱性氧 化物、酸性氧化物和同时具有酸碱性的两性氧化物. 研 究结果表明, 两性氧化物 $\left(\mathrm{CeO}_{2} 、 \mathrm{MnO}_{2} 、 \mathrm{La}_{2} \mathrm{O}_{3}\right)$ 的催化 活性要高于酸性氧化物和碱性氧化物. 
表 2 金属氧化物对乙酸酮基化反应活性影响 ${ }^{[59]}$

Table 2 Activity of $10 \mathrm{wt} \% \mathrm{MO}_{x} / \mathrm{SiO}_{2}$ catalysts in ketonization of acetic $\operatorname{acid}^{[59]}$

\begin{tabular}{|c|c|c|c|c|c|c|}
\hline \multirow{2}{*}{ Oxide } & \multicolumn{6}{|c|}{ Yield of acetone $/ \%$} \\
\hline & $300{ }^{\circ} \mathrm{C}$ & $325{ }^{\circ} \mathrm{C}$ & $350{ }^{\circ} \mathrm{C}$ & $375{ }^{\circ} \mathrm{C}$ & $400{ }^{\circ} \mathrm{C}$ & $425{ }^{\circ} \mathrm{C}$ \\
\hline $\mathrm{SiO}_{2}$ & 2 & 2 & 3 & 4 & 5 & 20 \\
\hline $\mathrm{B}_{2} \mathrm{O}_{3}$ & 2 & 2 & 2 & 2 & 3 & 6 \\
\hline $\mathrm{MoO}_{3}$ & 2 & 2 & 1 & 4 & 5 & 5 \\
\hline $\mathrm{WO}_{3}$ & 2 & 4 & 6 & 6 & 5 & 5 \\
\hline $\mathrm{P}_{2} \mathrm{O}_{5}$ & 1 & 1 & 6 & 10 & 12 & 9 \\
\hline $\mathrm{V}_{2} \mathrm{O}_{5}$ & 3 & 4 & 4 & 9 & 21 & 29 \\
\hline $\mathrm{Bi}_{2} \mathrm{O}_{3}$ & 10 & 6 & 5 & 11 & 18 & 28 \\
\hline $\mathrm{NiO}$ & 7 & 9 & 10 & 31 & - & - \\
\hline $\mathrm{Al}_{2} \mathrm{O}_{3}$ & 0 & 0 & 4 & 15 & 37 & 45 \\
\hline $\mathrm{CuO}$ & 5 & 5 & 6 & 29 & 39 & 43 \\
\hline $\mathrm{ZnO}$ & 6 & 9 & 10 & 19 & 33 & 54 \\
\hline $\mathrm{PbO}$ & 6 & 10 & 15 & 36 & 76 & 79 \\
\hline $\mathrm{Cr}_{2} \mathrm{O}_{3}$ & 1 & 8 & 52 & 48 & 39 & 46 \\
\hline $\mathrm{Fe}_{2} \mathrm{O}_{3}$ & 13 & 32 & 39 & 66 & 59 & 60 \\
\hline $\mathrm{CoO}$ & 13 & 15 & 48 & 50 & 63 & 64 \\
\hline $\mathrm{MgO}$ & 7 & 20 & 39 & 53 & 59 & 68 \\
\hline $\mathrm{Nd}_{2} \mathrm{O}_{3}$ & 3 & 3 & 6 & 22 & 61 & 70 \\
\hline $\mathrm{La}_{2} \mathrm{O}_{3}$ & 3 & 12 & 14 & 50 & 87 & - \\
\hline $\mathrm{MnO}_{2}$ & 18 & 22 & 34 & 72 & 96 & - \\
\hline $\mathrm{CdO}$ & 6 & 27 & 73 & 76 & 94 & - \\
\hline $\mathrm{CeO}_{2}$ & 9 & 24 & 31 & 96 & 97 & - \\
\hline
\end{tabular}

\section{3 催化剂氧化还原性影响}

除了对酸碱性的探究, 研究者们也研究了催化剂氧 化还原性对羧酸酮基化活性的影响, 尤其是 $\mathrm{CeO}_{2}$ 、 $\mathrm{TiO}_{2} 、 \mathrm{ZrO}_{2}$ 这些易被还原的氧化物. 经研究发现, 催化 剂经过还原后表面形成氧空位, 暴露了更多的配位不饱 和金属阳离子位, 去质子化后的羧化物更容易吸附在配 位不饱和金属阳离子位，有利于反应的进行.

Zaki 等 ${ }^{[60]}$ 研究了 $\mathrm{Al}_{2} \mathrm{O}_{3}, \mathrm{CeO}_{2}, \mathrm{TiO}_{2}$ 三种氧化物催化 乙酸酮基化反应. 这三种氧化物都具有 $\mathrm{L}$ 酸位, 但只有 $\mathrm{Al}_{2} \mathrm{O}_{3}$ 对乙酸酮基化反应没有催化活性, 究其原因是 $\mathrm{Al}_{2} \mathrm{O}_{3}$ 的阳离子不能被还原, 不能形成具有配位不饱和 金属阳离子位. Resasco 等 ${ }^{[19,30,41]}$ 研究了 $\mathrm{Ru} / \mathrm{TiO}_{2}$ 催化羧 酸酮基化反应. 不管是在液相反应还是气相反应中, 催 化剂经过还原后对反应的活性均有显著提高. 催化剂经 过 $\mathrm{H}_{2}$ 还原后, $\mathrm{Ti}^{4+}$ 被还原成 $\mathrm{Ti}^{3+}$, 形成了更多的氧空位、 暴露了更多的配位不饱和金属阳离子, 大大地提高了羧 酸的转化率. Simakova 等 ${ }^{[46]}$ 在 2013 年报道了反应气氛 对 $\mathrm{CeO}_{2} 、 \mathrm{ZrO}_{2} 、 \mathrm{CeO}_{2} / \mathrm{ZrO}_{2}$ 催化成酸酮基化反应活性的 影响, 比较了 $\mathrm{N}_{2}$ 和 $\mathrm{H}_{2}$ 对反应活性的影响(如表 3 所示),
通入氢气后催化剂被还原并暴露了更多的金属阳离子, 戊酸的转化率得到显著提高. 2014 年, Simakova 等 ${ }^{[61]}$ 又 报道了在 $\mathrm{H}_{2}$ 气氛下, $\mathrm{ZrO}_{2}$ 催化戊酸酮基化反应机理的 研究. 通过采用红外光谱和紫外-可见漫反射光谱 (UV-vis DRS)的表征手段，探究了 $\mathrm{ZrO}_{2}$ 对戊酸和 5-己酮 的吸附方式. 结合实验数据和 DFT 计算, 证明 $\mathrm{ZrO}_{2}$ 吸 附戊酸盐的方式取决于反应条件. 在 $\mathrm{H}_{2}$ 反应气氛下, 四 价锆阳离子 $\left(\mathrm{Zr}^{4+}\right)$ 可被还原成三价锆阳离子 $\left(\mathrm{Zr}^{3+}\right)$, 戊酸 在 $\mathrm{ZrO}_{2}$ 表面的存在方式可能是单分子单配位、单分子 桥连或是螯合配位，也可能是两个分子同时被吸附在一 个锆离子上. Overbury 等 ${ }^{[21]}$ 研究了 $\mathrm{CeO}_{2}$ (111) 晶面初始 氧化状态对乙酸反应的影响. 乙酸在 $\mathrm{CeO}_{2}$ (111) 晶面反 应生成物的脱附情况取决于催化剂表面的初始氧化状 态. 随着还原程度的增高, 形成的氧空位增多, 乙酸越 有利于进行单分子脱氧形成乙醛而不利于进行酮基化 形成丙酮. 适度还原催化剂有利于提高酮基化活性，但 过度还原则不利于酮基化反应. Pacchioni 等 ${ }^{[62]}$ 则通过密 度泛函理论计算研究 $\mathrm{ZrO}_{2}$ 预还原对乙酸酮基化反应的 影响. 在未还原的表面上, 去质子化后的乙酸盐进行烯 醇化过程需要较大的活化能, 并且很难进行脱氧形成乙 酰阳离子. 而 $\mathrm{ZrO}_{2}$ 经过 $\mathrm{H}_{2}$ 还原处理后, $\mathrm{Zr}^{3+}$ 周围的电子 很容易转移到乙酸盐离子上, 进行脱氧形成乙酰基, 再 与烯醇化合物反应生成 $\beta$-酮酸. 同时氧空位的存在也有 利于乙酰基中间物的形成.

表 $3 \mathrm{H}_{2}$ 和 $\mathrm{N}_{2}$ 对 $\mathrm{CeO}_{2} 、 \mathrm{ZrO}_{2} 、 10 \% \mathrm{CeO}_{2} / \mathrm{ZrO}_{2}$ 催化戊酸酮基化活性 的影响 ${ }^{[46]}$

Table 3 Catalytic properties of $\mathrm{CeO}_{2}, \mathrm{ZrO}_{2}$ and $10 \% \mathrm{CeO}_{2} / \mathrm{ZrO}_{2}$ oxides in the ketonization of valeric acid in hydrogen and nitrogen ${ }^{[46]}$

\begin{tabular}{|c|c|c|c|c|c|}
\hline \multirow{2}{*}{ Sample } & \multicolumn{2}{|c|}{ Conversion $/ \%$} & \multicolumn{2}{|c|}{ Selectivity/\% } & \multirow{2}{*}{$S /\left(\mathrm{m}^{2} \cdot \mathrm{g}^{-1}\right)$} \\
\hline & $\mathrm{H}_{2}$ & $\mathrm{~N}_{2}$ & $\mathrm{H}_{2}$ & $\mathrm{~N}_{2}$ & \\
\hline $\mathrm{CeO}_{2}$ & 40 & 31 & 76 & 80 & 25 \\
\hline $\mathrm{ZrO}_{2}$ & 83 & 66 & 78 & 80 & 105 \\
\hline $10 \%-\mathrm{CeO}_{2} / \mathrm{ZrO}_{2}$ & 93 & 82 & 79 & 81 & 79 \\
\hline
\end{tabular}

Reaction conditions: $355{ }^{\circ} \mathrm{C}, 1$ bar, $\mathrm{H}_{2}$ flow rate $30 \mathrm{~cm}^{3} \cdot \mathrm{min}^{-1}$, valeric acid LHSV $=0.34 \mathrm{~cm}^{3} \cdot \mathrm{g}^{-1} \cdot \mathrm{h}^{-1}$.

\section{4 结论与展望}

伴随着石化能源的消耗和环境污染的日益严重，生 物质能源作为一种可再生的清洁资源, 越来越引起研究 者们的注意. 通过一系列的反应过程可将木质纤维素转 化成油品和化学品, 来满足人们的需求. 羧酸在转化过 程中具有较高的含量, 所以将羧酸进行转化是非常关键 的一步. 研究发现, 通过酮基化反应将有机羧酸转化是 一条可行的方法, 但开发出具有低成本、高催化活性的 催化剂是在工业应用中要解决的重要的问题, 这也是我 们所面临的挑战与机遇. 金属氧化物催化羧酸酮基化反 应的机理与氧化物的晶格能量和反应温度等有关, 高晶 格能量的氧化物在高温下进行表面催化反应，在低温下 
进行体相反应. 当羧酸进行表面酮基化反应时，根据羧 酸分子的吸附情况不同生成的中间物不同, 可以分为 $\beta$ 酮酸机理和酮烯机理. 金属氧化物催化羧酸酮基化反应 中, 氧化物表面结构决定着酸的吸附方式和吸附能, 从 而影响反应活性. 氧化物酸碱性和还原性的改变直接影 响了表面结构的状态, 因此氧化物的酸碱性和还原性也 影响着酮基化反应的进行.

酤基化反应既可以在高温下发生气相反应，也可以 在低温下发生液相反应. 目前大多数研究者们关注于气 相酮基化反应, 对液相反应研究的较少. 生物质的衍生 物种类繁多、且难于分离, 易溶于水, 所以对酮基化液 相反应的研究显得更为有意义. 在酮基化反应的研究 中, 除了反应机理、催化剂种类及活性位的探索, 催化 剂的稳定性和失活的问题也是值得关注的. 不管在气相 反应还是在液相反应, 催化剂很容易失活. 造成失活的 原因很多, 如积碳、反应中间物堵塞活性位、溶剂的影 响等, 这都需要研究者们进行深入细致的研究.

羧酸酮基化反应属于表面结构敏感型反应，调控催 化剂的表面结构和性质可能会提高催化剂在温和条件 下的活性, 是未来值得研究的重要方向之一. 选用两性 金属氧化物作为主活性组分, 在此基础上掺杂或负载其 他金属元素, 来调控催化剂的表面结构(如酸碱对数量 和暴露金属阳离子的不饱和配位度)和性质(酸碱性和氧 化还原性), 有可能提高催化剂的反应活性, 进而使酮 基化反应在更温和条件下进行.

虽然酮基化反应是一个传统的化学反应, 但到目前 为止, 众多影响因素对酮基化反应活性的影响没有得到 统一的认知. 所以基于生物质能源的开发和利用, 对羧 酸酮基化反应深入细致的研究还是很有必要的, 且具有 重要的意义.

\section{作者简介}

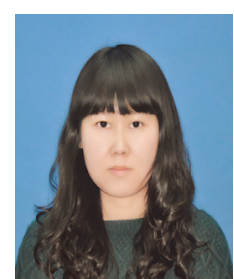

丁爽, 天津大学 2014 级在读博士生. 主要从事 Ce 基氧化 物催化羧酸酮基化反应机理研究.

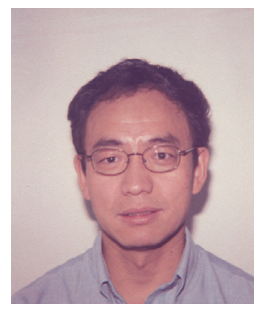

葛庆峰, 美国南伊利诺伊大学教授, 天津大学化工学院 教授, 博士生导师. 主要从事理论计算研究.

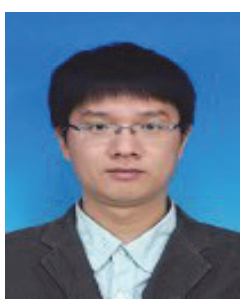

祝新利, 2007 毕业于天津大学获得博士学位, 2007 2011 年美国 Oklahoma 大学博士后研究助理及高级研究员, 2011 年 进入天津大学, 任副教授, 主要从事生物质催化转化研究.

\section{References}

[1] Huber, G. W.; Iborra, S.; Corma, A. Chem. Rev. 2006, 106, 4044

[2] Corma, A.; Iborra, S.; Velty, A. Chem. Rev. 2007, 107, 2411.

[3] Goyal, H. B.; Seal, D.; Saxena, R. C. Renew. Sust. Energ. Rev. 2008, $12,504$.

[4] Tao, J. J.; Chen, S.; Yao, F. Q.; Wang, H. H. Acta Chim. Sinica. 2016, 74, 81 (in Chinese). (陶骏骏, 陈帅, 姚奉奇, 王海晖, 化学 学报, 2016, 74, 81.)

[5] Ou, J. K.; Yang, L.; Xi, X. H. Chin. J. Chem. 2016, 34, 727.

[6] Dai, N.; Shang, R.; Fu, M. C.; Fu, Y. Chin. J. Chem. 2015, 33, 405.

[7] Li, J.; Huang, Y. B.; Guo, Q. X.; Fu, Y. Acta Chim. Sinica. 2014, 72, 1223 (in Chinese). (李江, 黄耀兵, 郭庆祥, 傅尧, 化学学报, 2014, 72, 1223.)

[8] Bertero, M.; Puente, G. D. L.; Sedran, U. Fuel. 2012, 95, 263.

[9] Ignatchenko, A. V.; Kozliak, E. I. ACS Catal. 2012, 2, 1555.

[10] Nagashima, O.; Sato, S.; Takahashi, R.; Sodesawa, T. J. Mol. Catal. A: Chem. 2005, 227, 231.

[11] Randery, S. D.; Warren, J. S.; Dooley, K. M. Appl. Catal., A 2002, $226,265$.

[12] Hendren, T. S.; Dooley, K. M. Catal. Today 2003, 85, 333.

[13] Dooley, K. M.; Bhat, A. K.; Plaisance, C. P.; Roy, A. D. Appl. Catal., A 2007, 320, 122

[14] Murkute, A. D.; Jackson, J. E.; Miller, D. J. J. Catal. 2011, 278, 189.

[15] Ignatchenko, A. V.; Deraddo, J. S.; Marino, V. J.; Mercado, A. Appl. Catal., A 2015, 498, 10.

[16] Friedel, C. Justus Liebigs Ann. Chem. 1858, 108, 122.

[17] Hu, M.; Zhu, Z. Q.; Xu, Z. H. Chem. Ind. Eng. Prog. 2010, 29, 316 (in Chinese). (胡永, 朱志庆, 徐泽辉, 化工进展, 2010, 29, 316.)

[18] Pestman, R.; Koster, R. M.; Duijne, A. V.; Pieterse, J. A. Z.; Ponec, V. J. Catal. 1997, 168, 265.

[19] Pham, T. N.; Shi, D.; Resasco, D. E. J. Catal. 2014. 314, 149.

[20] Pei, Z. F.; Ponec, V. Appl. Surf. Sci. 1996, 103, 171.

[21] Calaza, F. C.; Chen, T. L.; Mullins, D. R.; Xu, Y.; Overbury, S. H. Catal. Today 2015, 253, 65.

[22] Lee, Y.; Choi, J. W.; Suh, D. J.; Ha, J. M.; Lee, C. H. Appl. Catal., A $\mathbf{2 0 1 5}, 506,288$.

[23] Das, J.; Parida, K. React. Kinet. Catal. Lett. 2000, 69, 223.

[24] Parida, K.; Das, J. J. Mol. Catal. A: Chem. 2000, 151, 185.

[25] Martens, J. A.; Wydoodt, M.; Espeel, P.; Jacobs, P. A. Stud. Surf. Sci. Catal. 1993, 78, 527

[26] Gumidyala, A.; Sooknoi, T.; Crossley, S. J. Catal. 2016, 340, 76.

[27] Yamada, Y.; Segawa, M.; Sato, F.; Kojima, T.; Sato, S. J. Mol. Catal. A: Chem. 2011, 346, 79.

[28] Sun, C. H.; Chen, Y. S.; Li, J. Inorg. Chem. Ind. 2008, 40, 44 (in Chinese). (孙春晖, 陈永生, 李佳, 无机盐工业, 2008, 40, 44.)

[29] Pulido, A.; Oliver-Tomas, B.; Renz, M.; Boronat, M.; Corma, A. ChemSusChem 2013, 6, 141.

[30] Pham, T. N.; Dachuan, S.; Resasco, D. E. Top. Catal. 2014, 57, 706.

[31] Snell, R. W.; Shanks, B. H. ACS Catal. 2013, 3, 783.

[32] Gaertner, C. A.; Serrano-Ruiz, J. C.; Braden, D. J.; Dumesic, J. A. Ind. Eng. Chem. Res. 2010, 49, 6023.

[33] Gaertner, C. A.; Serrano-Ruiz, J. C.; Braden, D. J.; Dumesic, J. A. J. Catal. 2009, 266,71.

[34] Gaertner, C. A.; Serrano-Ruiz, J. C.; Braden, D. J.; Dumesic, J. A. ChemSusChem 2009, 2, 1121.

[35] Rajadurai, S. Catal. Rev. 1994, 36, 385.

[36] Renz, M. Eur. J. Org. Chem. 2005, 2005, 979.

[37] Ning, P. G.; Cao, H. B.; Zhang, Y. Mod. Chem. Ind. 2008, 28, 22 (in 
Chinese). (宁朋歌，曹宏斌，张懿，现代化工， 2008，28，22.)

[38] Pham, T. N.; Tawan, S.; Steven, P. C.; Resasco, D. E. ACS Catal. 2013, 3, 2456.

[39] Pacchioni, G. ACS Catal. 2014, 4, 2874

[40] Mekhemer, G. A. H.; Halawy, S. A.; Mohamed, M. A.; Zaki, M. I. J. Catal. 2005, 230, 109.

[41] Pham, T. N.; Shi, D.; Sooknoi, T.; Resasco, D. E. J. Catal. 2012, $295,169$.

[42] Idriss, H.; Diagne, C.; Hindermann, J. P.; Kiennemann, A.; Barteau, M. A. J. Catal. 1995, 155, 219.

[43] Zhang, Y.; Gao, Z. L.; Chen, Y. S.; Li, X. Chin. J. Rare Met. 2010, 34, 574 (in Chinese). (张义, 高中良, 陈永生, 李煦, 稀有金属, 2010, 34, 574.)

[44] Zhang, Y.; Gao, Z. L.; Chen, Y. S.; Li, X. Inorg. Chem. Ind. 2010, 4, 33 (in Chinese). (张义, 高中良, 陈永生, 李煦, 无机盐工业, 2010, 4, 33.)

[45] Gliński, M.; Zalewski, G.; Burno, E.; Jerzak, A. Appl. Catal., A 2014, 470, 278.

[46] Zaytseva, Y. A.; Panchenko, V. N.; Simonov, M. N.; Shutilov, A. A.; Zenkovets, G. A.; Renz, M.; Simakova, I. L.; Parmon, V. N. Top. Catal. 2013, 56, 846

[47] Crisci, A. J.; Dou, H.; Prasomsri, T.; Román-Leshkov, Y. ACS Catal. 2014, 4, 4196
[48] Snell, R. W.; Shanks, B. H. ACS Catal. 2014, 4, 512

[49] Teterycz, H.; Klimkiewicz, R.; Laniecki, M. Appl. Catal., A 2003, $249,313$.

[50] Kobume, M.; Sato, S.; Takahashi, R. J. Mol. Catal. A: Chem. 2008, $279,10$.

[51] Wang, W. D.; Lin, P. Y.; Fu, Y. L.; Yu, S. M.; Meng, M.; Zhang, X. P. Chin. J. Chem. 2000, $18,673$.

[52] Vohs, J. M. Chem. Rev. 2013, 113, 4136.

[53] Stubenrauch, J.; Brosha, E.; Vohs, J. M. Catal. Today 1996, 28,431.

[54] Wang, Z. L.; Feng, X. D. J. Phys. Chem. B 2003, 107, 13563.

[55] Snell, R. W.; Shanks, B. H. Appl. Catal., A 2013, 451, 86.

[56] Sun, J.; Gao, L. Acta Chim. Sinica 2002, 60, 1524 (in Chinese). (孙 静, 高濂, 化学学报, 2002, 60, 1524.)

[57] Kim, K. S.; Barteau, M. A. J. Catal. 1990, 125, 353.

[58] Ojamäe, L.; Aulin, C.; Pedersen, H.; Käll, P. O. J. Colloid Interface Sci. 2006, 296, 71.

[59] Glinski, M.; Kijenski, J.; Jakubowski, A. Appl. Catal., A 1995, 128 , 209.

[60] Hasan, M. A.; Zaki, M. I.; Pasupulety, L. Appl. Catal., A 2003, 243, 81

[61] Panchenko, V. N.; Zaytseva, Y. A.; Simonov, M. N.; Simakova, I. L.; Paukshtis, E. A. J. Mol. Catal. A: Chem. 2014, 388, 133.

[62] Tosoni, S.; Pacchioni, G. J. Catal. 2016, 344, 465.

(Cheng, B.; Fan, Y.) 